\title{
Relação entre o uso de inibidores de bomba de prótons e o desenvolvimento de demência senil: uma revisão de literatura
}

\author{
Relationship between the use of proton pump inhibitors and senil \\ dementia development: a literature review
}

\author{
1 Cristina Ribeiro Dias Barroso cristinavr95@hotmail.com \\ 1 Ticiani Rios Guedes \\ 1 Leonardo Silveira Gomes \\ 1 Vinícius Abrantes Silvestre \\ 1 Sérgio Elias Vieira Cury
}

1 UniFOA - Centro Universitário de Volta Redonda.

\section{Resumo}

Os inibidores da bomba de prótons (IBPs) representam uma das classes de medicamentos mais prescritas no mundo. No entanto, estudos recentes sugerem a relação entre seu uso crônico e o aumento do risco para o desenvolvimento de demência senil (DS). Este artigo objetiva analisar a relação entre o uso prolongado dos IBPs e o aumento do risco para o desenvolvimento de DS, baseado na revisão de literatura recente sobre o assunto. Foi realizada uma revisão bibliográfica com base em 36 artigos científicos encontrados nas bases de dados do PubMed/MEDLINE e SciELO. Entre eles, três importantes estudos de 2013, 2015 e 2016 evidenciaram forte relação entre a população idosa em uso de IBPs e o aumento do desenvolvimento de DS. Concluiu-se que estudos prospectivos conseguiram comparar o uso crônico dos IBPs e a incidência de DS nos pacientes usuários versus o não uso desses medicamentos, revelando que sua incidência quase dobrou nos pacientes que fizeram uso crônico da medicação.

\section{Palavras-chave}

Omeprazol. Doença de Alzheimer. Demência.

\begin{abstract}
Proton pump inhibitors (PPIs) represent one of the most prescribed classes of medicines in the world. However, recent studies suggest the relationship between their chronic use and the increased risk for the development of senile dementia (SD). This article aims to analyze the relationship between the prolonged use of PPIs and the increased risk for the development of DS, based on a review of recent literature on the subject. A bibliographic review was performed based on 36 scientific articles of PubMed/MEDLINE and SciELO databases. Among them, three important studies of 2013, 2015 and 2016 evidenced a strong relationship between the elderly population using PPIs and the increased $S D$ development. It was concluded that prospective studies were able to compare the chronic use of PPIs and the incidence of SD in patients that were using the medication versus the non-use of these drugs, revealing that their incidence almost doubled in patients with chronic use.
\end{abstract}

\section{Keywords}

Omeprazole. Alzheimer's disease. Dementia.

\section{Como você deve citar?}

BARROSO, Cristina Ribeiro Dias et al. Relação entre o uso de inibidores de bomba de prótons e o desenvolvimento de demência senil: uma revisão de literatura. Cadernos UniFOA, Volta Redonda, n. 37, p. 137-146, ago. 2018. 


\section{INTRODUÇÃO}

Os inibidores da bomba de prótons (IBPs) representam uma das classes de medicamentos mais prescritas no mundo, pois combinam um alto nível de eficácia juntamente com uma baixa toxicidade (FORGACS; LOGANAYAGAM, 2008). Introduzidos na década de 1980, tais medicações são utilizadas para tratar os distúrbios relacionados ao aumento da acidez do trato gastrointestinal superior ao inibir a secreção gástrica. Segundo estudos do National Health Interview Survey, o uso de IBPs aumentou de $3,4 \%$ para $7,0 \%$ entre os homens e de $4,8 \%$ para $8,5 \%$ entre as mulheres, de 2000 a 2013 (LEWIS, 2016).

Acredita-se que os IBP possam atravessar a barreira hematoencefálica, aumentando tanto a produção quanto a degradação amiloide, além de se ligarem à TAU (proteína intimamente relacionada ao desenvolvimento da Doença de Alzheimer (DA)). Há também evidências da redução dos níveis de vitamina $\mathrm{B} 12$ e outros nutrientes entre os pacientes que fazem uso dos IBP, fatores que podem estar relacionados com um risco aumentado de demência nesses pacientes (KULLER, 2016).

A vitamina B12 é importante para a formação e maturação das hemácias, além de ser necessária para o desenvolvimento e manutenção das funções do sistema nervoso. Sua principal fonte é através da ingestão de alimentos de origem animal. No entanto, para absorvê-la, o corpo depende de fatores intrínsecos presentes em um tipo especial de células do estômago (as células parietais) e de receptores localizados no íleo, estruturas nas quais os inibidores de bomba proteônica causam alterações e diminuem suas funções absortivas (LAM, 2013).

A Demência Senil (DS) é uma doença mental progressiva, caracterizada por um prejuízo cognitivo que pode incluir alterações de memória, raciocínio, concentração, aprendizado, realização de tarefas complexas, julgamento, linguagem e habilidades, além de desorientação em relação ao tempo e ao espaço. Ela tem um peso substancial e crescente sobre os pacientes, suas famílias e o sistema de saúde (PRINCE, 2013).

Ademais, a alta demanda em terapias e cuidados por causa do declínio cognitivo cumulativo, combinado ao aumento do número de pacientes portadores de demência possui um notável impacto socioeconômico. A estimativa em nível mundial dos custos relacionados à demência foi de US\$ 604 bilhões, no ano de 2010 (NITRINI, 2013).

Dessa forma, torna-se imprescindível o conhecimento, principalmente por parte dos médicos, da relação existente entre a utilização crônica dos medicamentos inibidores da bomba de prótons e o aumento do risco para o desenvolvimento de DS, uma vez que a doença não apresenta somente repercussões clínicas nos pacientes portadores, mas também sociais e econômicas para todo o sistema de saúde do país.

\section{MATERIAIS E MÉTODOS}

Foi realizada uma revisão bibliográfica através de pesquisas nas bases de dados PubMed/MEDLINE e SciELO. As buscas na base PudMed/MEDLINE foram realizadas em língua inglesa, sendo utilizados os seguintes descritores: "Omeprazole"; "Proton pump inhibitors"; "Senile Dementia"; "Alzheimer's Disease"; "Vascular Dementia"; "Frontotemporal Lobe Dementia FLDEM"; "Lewy Body Dementia". As pesquisas realizadas na base de dados SciELO utilizaram os mesmos descritores apresentados em língua portuguesa. 
Foram analisados artigos de relevância científica, dentre eles, revisões bibliográficas e estudos coorte. Os estudos selecionados abordaram os seguintes temas: o mecanismo de ação, a utilização clínica e as principais alterações orgânicas constatadas com o uso de IBPs, os diferentes tipos de DS e a relação entre o uso crônico dos IBPs e o aumento do risco de desenvolvimento de DS. Os artigos que não abordaram nenhum dos temas acima foram excluídos da pesquisa.

Após extensa leitura, foram selecionados 36 artigos científicos. Ademais, também foi utilizado o livro "Harrison: Medicina Interna - 16 a edição" e dados de pesquisas da plataforma IBGE e National Health Interview Survey.

\section{RESULTADOS}

\subsection{Os inibidores de bomba de prótons (IBPs)}

Os inibidores da bomba de prótons (IBPs) - omeprazol, pantoprazol, lansoprazol, rabeprazol, esomeprazol e tenatoprazol - suprimem a secreção de ácido gástrico. Embora haja diferenças farmacocinéticas, todos os representantes dessa classe são similares entre si, reduzindo em até $95 \%$ a produção diária de ácido gástrico (BADIOLA, 2013).

Segundo Park (2014), a supressão ácida exerce papel essencial no manejo de doenças pépticas relacionadas à acidez gástrica. Os IBPs apresentam eficácia definida no tratamento de manifestações e complicações de doença péptica, doença do refluxo gastresofágico (tratamento de escolha), tratamento sintomático e cicatrização de úlceras pépticas induzidas por uso crônico de anti-inflamatórios não esteroides (AINE), prevenção de lesões gastrintestinais induzidas por uso crônico de AINE e coadjuvante na redução de recorrência de úlceras pépticas induzidas por Helicobacter pylori (STEDMAN, 2013).

No que diz respeito ao mecanismo de ação, assim como afirmado por Yu (2015), Maximov, em 2013, também já havia mencionado que a secreção ácida nas células parietais do estômago cria um gradiente de íons, em que prótons são bombeados de fluidos intracelulares para o lúmen gástrico contra esse gradiente, sendo a bomba $\mathrm{H}+, \mathrm{K}+$ ATPase responsável por esse transporte ativo.

Além disso, Nishida (2014) também complementa a literatura, ao afirmar que a bomba $\mathrm{H}+, \mathrm{K}+$ ATPase está localizada, na sua forma inativa, na membrana dos túbulos citoplasmáticos e, quando ativada, localiza-se na membrana dos canalículos da célula parietal do estômago. Ademais, tal bomba é composta de duas subunidades polipeptídicas não idênticas, a catalítica ou "a" e a estrutural ou "b". Sua atividade fisiológica da bomba $\mathrm{H}+, \mathrm{K}+$ ATPase está sujeita à modulação (estimulação e inibição) hormonal e por neurotransmissores, tais como somastatina, gastrina, acetilcolina e histamina (TAMELINI; THOMSON, 2013).

Ainda sobre os neurotransmissores, o mesmo estudo já citado revelou que a acetilcolina é liberada no plasma sanguíneo por fibras nervosas pós-ganglionares, e a gastrina, através do estímulo propiciado pela alimentação (KUIPERS, 2006). Além disso, ambas podem ativar a secreção ácida indiretamente, estimulando a liberação de histamina das células enterocromafins e, diretamente, atuando em receptores da célula parietal (WANNMACHER, 2014).

Tolman (2012) e Novotna (2014) também afirmam que a inibição da secreção ácida ocorre por mecanismo de retroalimentação, que previne excesso de conteúdo ácido. Quando o pH gástrico é menor que 2,5, sobrevém a liberação de somastatina no plasma, bloqueando a secreção de gastrina e aumentando o $\mathrm{pH}$. 


\subsection{Aplicabilidade clínica}

No ano de 2013, estudos de Maximov revelaram que os IBPs são instáveis em meio ácido e, por isso, quando administrados oralmente, são revestidos para proteção contra a rápida degradação no estômago, sendo absorvidos no duodeno. As apresentações farmacêuticas disponíveis no mercado são: injetáveis, comprimidos com revestimento entérico de liberação retardada, comprimidos de desintegração rápida, cápsulas com grânulos com revestimento entérico de liberação normal e retardada.

É importante destacar que os IBPs são pró-drogas e devem ser administrados antes da ingestão de alimentos. Estes últimos estimularão a produção de gastrina, a qual, por sua vez, ativará a bomba $\mathrm{H}+, \mathrm{K}+$ ATPase, presente nos canalículos da célula parietal, proporcionando um ambiente ácido $(\mathrm{pH} \sim 1)$, onde, a seguir, ocorrerá a ativação da pró-droga. Essa etapa assegura que, após a absorção, a partir da corrente sanguínea, o IBP passe para as células parietais, onde será ativado. Os IBPs formam uma ligação covalente, provocando a inibição irreversível da bomba, permitindo, assim, exercer seu efeito (SODHI, 2013).

O mesmo estudo realizado por Sodhi e colaboradores mostra que os IBPs inibem a secreção ácida basal e a estimulada por alimentos e mediadores neuronais já mencionados. Após a alimentação, apenas as bombas ativadas na célula parietal são inibidas pelos IBPs. A meia-vida da bomba $\mathrm{H}+, \mathrm{K}+$ ATPase é de 50 horas e, a cada 24 horas, cerca de $25 \%$ de novas bombas são sintetizadas. A restauração da secreção ácida geralmente ocorre após 72 horas da suspensão do IBP, por meio da síntese de novas bombas protônicas e ativação de bombas que estavam internalizadas em estado inativo.

Os anti-inflamatórios são largamente utilizados no tratamento de doenças osteoarticulares. Seu mecanismo de ação básico é a inibição da prostaglandina, elemento importante na produção da barreira mucosa gástrica que protege a mucosa do estômago (WILLIANMS, 2012). Quando essa barreira é quebrada, ocorre a retro difusão de íons hidrogênio que induzem a lesão da camada mucosa pela produção de espécies reativas do oxigênio (radicais livres) (GOYAL, 2012). 0 resultado final é o desenvolvimento de gastrite erosiva e úlcera gástrica e, em menor escala, a úlcera duodenal. Os IBPs assumem papel destacado na prevenção e cicatrização dessas lesões, em função do ser um potente inibidor da secreção ácida (PARK, 2014).

A síndrome de Zollinger-Ellison é doença rara causada por tumor secretor de gastrina que resulta em hipersecreção ácida maciça no estômago que, secundariamente, levará à formação de úlceras pépticas e diarreia (SOHAILY, 2013). Antes do advento dos IBPs, pacientes portadores dessa síndrome tinham como medida terapêutica principal a gastrectomia total. $\mathrm{Na}$ atualidade, o tratamento clínico desses pacientes tem como pedra angular o uso de IBPs, no entanto, em doses bem maiores que aquelas habitualmente utilizadas para tratamento de outras enfermidades ácido-pépticas, podendo ser utilizadas doses iniciais de até $160 \mathrm{mg} /$ dia para protegê-los das graves e potencialmente letais complicações pépticas que estão relacionadas à hipersecreção ácida no estômago (FAWAS, 2014).

O efeito dos IBPs é dose-dependente e a rapidez para alcançar o pico de ação depende da sua biodisponibilidade, ou seja, a taxa e o grau com que é absorvido e se torna disponível no sítio de ação (US Food and Drug Administration, 2003). Além disso, segundo Poitras e colaboradores (2012), essa biodisponibilidade tende a aumentar com o tempo de tratamento com omeprazol e esomeprazol, não sendo observada essa característica com os demais IBPs.

Estudo de Alai (2014) mostrou que outra aplicação clínica importante dos IBPs é como adjuvante no tratamento de erradicação do Helicobacter pylori. Uma metanálise recente que envolveu 35 estudos e 5.998 pacientes comparou o uso de esomeprazol ou rabeprazol com outros IBPs (omeprazol, 
lanzoprazol, pantoprazol) no tratamento de erradicação do $H$. pylori e concluiu que os pacientes que fizeram uso do esomeprazol ou rabeprazol nos seus tratamentos alcançaram maior taxa de erradicação, sobretudo no subgrupo de pacientes classificados como metabolizadores rápidos. Os IBPs se acham também indicados no tratamento das úlceras pépticas.

\subsection{Uso crônico dos IBPs e suas complicações}

Há situações crônicas que justificam o uso contínuo de IBPs, porém, para a maioria dos pacientes cujos sintomas gastrintestinais são persistentes, é possível o tratamento intermitente com tal medicamento. As situações clínicas que podem justificar o uso contínuo de IBPs são: doença do refluxo gastresofágico, esôfago de Barret e úlceras induzidas por AINE, segundo Poitras, em 2012, e Alai, em 2015.

A Doença do Refluxo Gastresofágico é um dos distúrbios gastrintestinais mais prevalentes. Estudos populacionais mostram que até $15 \%$ dos indivíduos apresentam pirose e/ou regurgitação, pelo menos uma vez por semana, e 7\%, diariamente (FALLAHZADEH, 2015). Os IBPs são mais eficazes no alívio dos sintomas e mais comumente usados. $O$ tratamento durante oito semanas promove cicatrização da esofagite erosiva, em 90\% dos casos (YU, 2015).

O Esôfago de Barrett é uma complicação grave da esofagite por refluxo e fator de risco para adenocarcinoma esofágico (MOWAT, 2012). Recomenda-se o uso contínuo de um IBP em dose única diária. Pacientes que não respondem clinicamente ao tratamento podem tomar o IBP em duas doses diárias. No entanto, não há provas de que o tratamento de supressão ácida com os IBPs promova regressão do Esôfago de Barrett ou impeça a progressão da doença para adenocarcinoma do esôfago (YU, 2015).

Pacientes com úlceras induzidas por anti-inflamatório não esteroide (AINE) devem suspender ou reduzir a dose do AINE e receber tratamento de erradicação do Helicobacter pylori, se esta estiver presente. Quando for inviável alterar o tratamento com AINE, recomenda-se adicionar um antissecretor (ALAI, 2015).

Os IBPs são bem tolerados e, na maioria dos casos em que surgem efeitos adversos, estes são moderados e passageiros. Os mais comuns, sendo observados em aproximadamente $10 \%$ dos pacientes, são: cefaleia, diarreia, distúrbios gastrintestinais, constipação e flatulência (MACKAY, 2014). Efeitos adversos raros, porém importantes, incluem nefrite aguda intersticial, hiponatremia, hipopotassemia, hipomagnesemia, pancreatite e síndrome de Stevens-Johnson (ALAI, 2015).

Os IBPs diminuem a absorção de vitamina B12 ao reduzirem a acidez gástrica. Essa ação se deve a uma alteração no ciclo do pepsinogênio, o qual necessita de certa acidez gástrica para ser transformado em pepsina, retirando, assim, a vitamina B12 contida nos alimentos ingeridos (MCCOLL, 2012). Em pacientes idosos que já possuem atrofia gástrica, possivelmente por infecção de $H$. pylori, o uso crônico de IBP pode reduzir a concentração sérica de vitamina B12, segundo Sahara e colaboradores (2013).

No trabalho de Fang (2014), estudos sugerem que somente pacientes com intensa supressão ácida por vários anos, como no tratamento da Síndrome de Zollinger-Ellison, estão em risco de desenvolverem deficiência relevante de vitamina B12 e estes são os que devem ser monitorados. Além disso, estudos anteriores de Sharma, em 2014, complementam tal preposição, ao afirmarem que a absorção duodenal de ferro orgânico e não orgânico também pode ser afetado com o tratamento crônico desses medicamentos. 


\subsection{Demência Senil}

A demência é uma doença progressiva caracterizada pela deterioração da capacidade cognitiva e redução da capacidade para uma vida independente $(\mathrm{HAENISCH}, 2015)$. Tem peso substancial e crescente sobre os pacientes, suas famílias e o sistema de saúde. As análises de dados a partir dos fundos previdenciários alemães mostrou que a prevalência de demência é de cerca de $1 \%$ na idade de 65 anos e aumenta com a idade. Aos 90 anos, cerca de um terço da população é afetada, segundo estudos de Fang (2014).

Segundo Rouch (2015), a previsão é que a prevalência global de demência aumentará de cerca de 35 milhões para mais de 80 milhões de pacientes em todo o mundo em 2040. A alta demanda de terapias e cuidados por causa do declínio cognitivo, combinado com um número crescente de pacientes, tem um impacto socioeconômico notável. Os custos em todo o mundo estimados por demência foram de US\$ 604 bilhões, no ano de 2010 (MOHER, 2014). Visando reduzir o grande contingente de pacientes afetados, sistemas de saúde de todo o mundo deverão trabalhar para medidas de prevenção sobre a população em maior risco, os idosos. Por isso, estudos epidemiológicos que analisem a utilização de medicamentos potencialmente deflagradores da DS tornam-se extremamente relevantes (ROUCH, 2015).

\subsection{Relação entre o uso crônico de IBPs e o aumento do risco de desenvolvimento de Demência Senil}

Em estudo realizado por Haenisch e colaboradores (2015), em que foram acompanhados 3.327 idosos com idade igual ou superior a 75 anos, por um intervalo de 18 meses, foi comparado o efeito do uso crônico dos IBPs (omeprazol, esomeprazol, lansoprazol, pantoprazol, rabeprazol e dexlansoprazol) sobre a incidência de demência versus a não utilização dessas drogas. A coleta de dados foi realizada por médicos e psicólogos treinados, através de prontuários, história clínica e realização de testes cognitivos baseados na Entrevista Estruturada para Diagnóstico de Demência do tipo Alzheimer, Multiinfarte e demências de outras etiologias (55 testes cognitivos, incluindo 30 itens do MiniMental, Exame de Estado, e avaliação de atividades diárias - Escala ADL SIDAM), além da Escala de Hachinski-Rosen.

Ao final do estudo, obteve-se como resultado um total de 431 pacientes com qualquer incidência de demência, incluindo 260 doentes com diagnóstico de DA. Considerados os dados pertinentes, além de itens considerados variantes de confusão (como sexo, idade, presença de predisposição genética para a doença, cardiopatia ou doença isquêmica cerebral), concluiu-se que os pacientes que fizeram uso regular de algum dos IBPs mostraram um aumento significativo do risco de desenvolver qualquer demência [razão de Hazard (HR) 1,38, 95\% de intervalo de confiança (IC) 1,04-1,83] e DA (HR 1,44 , IC de $95 \%$ 1,01-2,06), comparado aos não usuários.

Posteriormente, no ano de 2016, Gomm e equipe realizou um estudo prospectivo de coorte, usando dados observacionais de 2004 a 2011, derivados da maior seguradora de saúde legal alemã, a Allgemeine Ortskrankenkassen (AOK). Os dados utilizados estavam disponíveis em uma base trimestral, onde foram analisados pacientes internados e aqueles diagnosticados ambulatorialmente (dados codificados pela Classificação Estatística Internacional de Doenças e Problemas Relacionados à Saúde, após modificação alemã), incluindo suas prescrições de medicamentos (categorizados de acordo com o Sistema de Classificação Anatomical Therapeutic Chemical). A análise dos dados foi realizada de agosto a novembro de 2015.

O resultado desse estudo foi composto pela análise de um total de 73.679 participantes de 75 anos de idade ou mais velhos, e livres de demência no início do estudo. Os pacientes que receberam a medicação IBP normal ( $n=2.950$; média [SD] idade, 83,8 [5,4] anos; $77,9 \%$ do sexo feminino) apre- 
sentaram um risco significativamente aumentado de demência em comparação com os pacientes que não receberam tal medicação ( $n=70729$; média [SD] idade, 83,0 [5,6] anos; 73,6\% do sexo feminino) (hazard ratio, [95\% IC, 1,36-1,52] 1,44; $\mathrm{P}<.001$ ).

Segundo a pesquisa, a evasão da medicação pode impedir o desenvolvimento de demência. Essa conclusão é corroborada pelas recentes análises em dados farmacoepidemiológicos primários e está em consoante com modelos de ratos, nos quais a utilização dos IBPs aumentou os níveis de $\beta$-amiloide no cérebro desses animais. Nesse momento, mais ensaios clínicos prospectivos randomizados são necessários para examinar essa conexão com mais detalhes.

Nesse contexto, vale ressaltar o estudo realizado por Badiola e sua equipe, em 2013. Tal estudo comprovou o aumento da produção de substâncias amiloides, altamente relacionada à ocorrência da demência, incluindo a DA, após utilização prolongada de IBPs (sendo utilizado o medicamento lanzoprazol). A pesquisa foi baseada na aplicação do IBP, seguido da produção de anticorpos em modelos celulares e animais, tendo o posterior desenvolvimento da Doença de Alzheimer. Verificou-se que o lansoprazol aumentou a produção das proteínas $A \beta 37, A \beta 40$ e $A \beta 42$, considerados peptídeos amiloides.

Assim, segundo o estudo, o tratamento agudo com lansoprazol em ratos do tipo selvagem e transgênicos promoveu níveis mais elevados de $A \beta 40$ no cérebro desses animais, o que pode indicar que o potencial de tal medicamento exacerbar também a produção de proteínas $A \beta$ no cérebro de seres humanos e, consequentemente, aumentar a incidência de Doença de Alzheimer.

\section{CONCLUSÃO}

Estudos prospectivos realizados conseguiram comparar o efeito do uso crônico dos IBPs sobre a incidência de DS versus o não uso desses medicamentos, revelando que a incidência de DS quase dobrou nos pacientes que usaram a medicação por longo prazo. Assim, torna-se imprescindível o maior conhecimento médico sobre o risco do uso crônico de tais drogas, sendo necessária uma análise mais criteriosa quanto a sua real necessidade de utilização. No entanto, o desenvolvimento de mais estudos acerca desse assunto é fundamental para aumentar o grau de evidências das pesquisas existentes e ampliar os fundamentos sobre tal relação. 


\section{REFERÊNCIAS}

ALAI, M.; LIN, W.J. Application of nanoparticles for oral delivery of acid-labile lansoprazole in the treatment of gastric ulcer: in vitro and in vivo evaluations. Int J Nanomedicine, v. 18, n. 10, p. 4029-41, 2015.

ALAI, M.; LIN, W.J. Novel lansoprazole-loaded nanoparticles for the treatment of gastric acid secretionrelated ulcers: in vitro and in vivo pharmacokinetic pharmacodynamic evaluation. AAPS J, v. 16, n. 3 , p. 361-72, 2014.

BADIOLA, N.; ALCALDE, V.; PUJOL, A. et al. The Proton-Pump Inhibitor Lansoprazole Enhances Amyloid Beta Production. PLoS one, v. 8, p. 1-8, 2013.

FALLAHZADEH, M. K.; BORHANI HAGHIGHI, A.; NAMAZI, M. R. Proton pump inhibitors: predisposers to Alzheimer disease? Journal of clinical pharmacy and therapeutics, v. 35, n. 2, p. 125-126, 2015.

FANG, Y.; WANG, G.; ZHANG, R. et al. Eudragit L/HPMCAS blend enteric-coated lansoprazole pellets: enhanced drug stability and oral bioavailability. AAPS Pharm Sci Tech, v. 15 n. 3, p. 513-21, 2014.

FAWAZ, M.V.; BROOKS, A.F.; RODNICK, M.E. et al. High affinity radiopharmaceuticals based upon lansoprazole for PET imaging of aggregated tau in Alzheimer's disease and progressive supranuclear palsy: synthesis, preclinical evaluation, and lead selection. ACS Chem Neurosci, v. 5, n. 8, p. 718-30, 2014.

FORGACS, I.; LOGANAYAGAM, A. Overprescribing proton pump inhibitors. BMJ: British Medical Journal, v. 336, n. 7634, p. $2,2008$.

GOMM, W.; VON HOLT, K.; THOMÉ, F. et al. Association of Proton Pump Inhibitors With Risk of Dementia - A Pharmacoepidemiological Claims Data Analysis, JAMA Neurol, v .10, n. 1001, p. 1-7, 2016.

GOYAL, R.K. Distúrbios do Sistema Gastrintestinal: Doenças do esôfago. In: KASPER, D.L.; FAUCl, A.S.; LONGO, D.L., et al. Harrison: Medicina Interna. 16. ed. Rio de Janeiro: Mc Graw Hill, 2012.

HAENISCH, B.; VANS HOLT, K.; WIESE, B. et al. Risk of dementia in elderly patients with the use of proton pump inhibitors. Eur Arch Psychiatry Clin Neurosci, 2015.

INSTITUTO BRASILEIRO DE GEOGRAFIA E ESTATÍSTICA - IBGE. Disponível em: <http://www.ibge.gov. br/home/>. Acesso em: 15 dez 2016.

KUIPERS, E.J. Proton pump inhibitors and gastric neoplasia. Gut, v. 55, p. 1217-21, 2006.

KULLER, L.H. Do proton pump Inhibitors Increase the risk of dementia?. JAMA neurology, v. 73, n. 4, p. 379-381, 2016.

LAM, J.R. et al. Proton pump inhibitor and histamine 2 receptor antagonist use and vitamin B12 deficiency. Jama, v. 310, n. 22, p. 2435-42, 2013.

LEWIS, H.K. Do Proton Pump Inhibitors Increase the Risk of Dementia? JAMA Neurol, 2016.

MACKAY, J.D.; BLADON, P.T. Hypomagnesemia due to proton-pump inhibitor therapy: a clinical case series. Q J Med, v. 103, p. 387-95, 2014. 
MAXIMOV, G.; KAMNASARAN, D. The adjuvant use of lansoprazole, clonazepam and dimenhydrinate for treating intractable hiccups in a patient with gastritis and reflux esophagitis complicated with myocardial infarction: a case report., DBMC Res Notes, v. 16, n. 6, p. 327, 2013.

MCCOLL, K.E.L.; KENNERLEY, P. Proton pump inhibitors- differences emerge in hepatic metabolism. Digest Wjer Dis, v. 34, p. 461-7, 2012.

MOHER, D.; LIBERATI, A.; TETZLAFF, J. et al. Preferred reporting items for systematic reviews and metaanalyses: the PRISMA statement. BMJ. 2014.

MOWAT, C.; WILLIAMS, C.; GILLEN, D. et al. Omeprazole, Helicobacter pylori status and alterations in the intragastric milieu facilitating bacterial N-nitrosation. Gastroenterology, v. 119, n. 339-47, 2012.

NISHIDA, T.; TSUJII, M.; TANIMURA, H. et al. Comparative study of esomeprazole and lansoprazole in triple therapy for eradication of Helicobacter pylori in Japan. World J Gastroenterol, v. 20, n. 15, p. 4362-9, 2014.

NITRINI, R.; CARAMELLI, P.; BOTTINO, C.M.C. et al. Diagnóstico de doença de Alzheimer no Brasil. Arq Neuropsiquiatr, v. 63, n. 3A, p. 720-7, 2013.

NOVOTNA, A.; SROVNALOVA, A.; SVECAROVA, M. et al Differential effects of omeprazole and lansoprazole enantiomers on aryl hydrocarbon receptor in human hepatocytes and cell lines. PLoS one, v. 9, n. 6, 2014.

PARK, C.H.; KIM, E.H.; ROH, Y.H. et al. The association between the use of proton pump inhibitors and the risk of hypomagnesemia: a systematic review and meta-analysis., Journal Pone, v. 9, n. 11, 2014.

POITRAS, P.; GINGRAS, M.H.; REHFELD, J.F. The Zollinger-Ellison syndrome: dangers and consequences of interrupting antisecretory treatment. Clin Gastroenterol Hepatol, v. 10, p. 199-202, 2012.

PRINCE, M. Bryce, R.; Albanese, E. et al. The global prevalence of dementia: a systematic review and metaanalysis. Alzheimer's \& Dementia, v. 9, n. 1, p. 63-75, 2013.

ROUCH, L.; CESTAC, P.; HANON, O. et. al. Antihypertensive Drugs, Prevention of Cognitive Decline and Dementia: A Systematic Review of Observational Studies, Randomized Controlled Trials and MetaAnalyses, with Discussion of Potential Mechanisms, CNS Drugs, v. 29, n. 2, p. 40-52, 2015.

SAHARA, S.; SUGIMOTO, M.; UOTANI, T. et al. Twice-daily dosing of esomeprazole effectively inhibits acid secretion in CYP2C19 rapid metabolisers compared with twice-daily omeprazole, rabeprazole or lansoprazole. Aliment Pharmacol Ther, v. 38, n. 9, p. 1129-37, 2013.

SHARMA, V.R.; BRANNON, M.A.; CARLOSS, E.A. Effect of omeprazole on oral iron replacement in patients with iron deficiency anemia. Southern medical journal, v. 97, n. 9, p. 887-889, 2014.

SODHI, R.K.; SINGH, N. Defensive effect of lansoprazole in dementia of AD type in mice exposed to streptozotocin and cholesterol enriched diet. PloS one, v. 8, n. 7, 2013.

SOHAILY, S.A.; DUGGAN, A. Long term management of patients taking proton pump inhibitors, Australian Prescriber, Newcastle, n. 31, p. 5-7, 2013. 
STEDMAN, C.A.M.; BARCLAY, M.L. Comparison of the pharmacokinetics, acid suppression and eficacy of proton pump inhibitors. Aliment Pharmacol Ther, v.14, p. 963-78, 2013.

TAMELINI, M.G.; FORLENZA, O.V. Diagnóstico diferencial das demências. Rev Psiq Clín, v. 32, n. 3, p. 11930, 2013.

THOMSON, A.B.; SAUVE, M.D.; KASSAM, N. et al. Safety of the long-term use of proton pump inhibitors. World Journal of Gastroenterology, v. 16, n. 19, p. 2323-30, 2013.

TOLMAN, K.G.; SANDERS, S.W.; BUCHI, K.N. et al. The effects of oral doses of lansoprazole and omeprazole on gastric pH. Journal of Clinical Gastroenterology, v. 24, n. 2, p. 65-70, 2012.

US FOOD AND DRUG ADMINISTRATION. Guidance for industry bioavailability and bioequivalence studies for orally administered drug products - General considerations USA. 2003. Disponível em: <http://www. fda.gov/cder/guidance/5356fnl.pdf>. Acesso em: 20 set 2016.

WANNMACHER, L. Inibidores da bomba de prótons: indicações racionais. Uso Racional de Medicamentos Temas Selecionados, Brasília, v. 2, n. 1, p. 1-6, 2014.

WILLIANMS, C.; MCCOLL, K.E.L. Proton pump inhibitors and bacterial overgrowth. Alim Pharm \& Therap, v. 23, p. 3-10, 2012.

YU, M.; LEE, C.; WANG, M.; et al. Influence of the proton pump inhibitor lansoprazole on distribution and activity of doxorubicin in solid tumors. Cancer Sci, v. 106, n. 10, p. 1438-47, 2015. 